\title{
An Analysis of the Risk Factors for New-Onset Diabetes Mellitus After Liver Transplantation
}

\author{
Jushan Sun' \\ Yibiao $\mathrm{He}^{1}$ \\ Lei Bai' \\ Zhipeng Wang' \\ Zhu Cao' \\ Yingmei Shao ${ }^{2}$ \\ Jinming Zhao' \\ 'Department of Liver and Laparoscopic \\ Surgery, Digestive and Vascular Surgery \\ Center, The First Affiliated Hospital of \\ Xinjiang Medical University, Urumqi, \\ Xinjiang, 830054, People's Republic of \\ China; ${ }^{2}$ Xinjiang Uyghur Autonomous \\ Region Clinical Research Center for \\ Echinococcosis and Hepatobiliary \\ Diseases, The First Affiliated Hospital of \\ Xinjiang Medical University, Urumqi, \\ Xinjiang, 830054, People's Republic of \\ China
}

Objective: To investigate the risk factors related to new-onset diabetes mellitus (NODM) and the significance of IL-6.

Methods: A retrospective analysis was conducted on clinical data from 64 patients who received either a living donor liver transplantation or a donation after circulatory death from September 2013 to October 2020 and attended regular follow-up visits for six or more months. During follow-up, patients were randomized into groups and followed up until the completion of the study or the death of the patient.

Results: The incidence of NODM was $31.25 \%(n=20)$. The median age in the NODM group was 52.15 years $(\mathrm{p}<0.01)$. Age $(\mathrm{OR}=1.089 ; 95 \% \mathrm{CI}: 0.0211-0.1495, \mathrm{p}=0.003)$ and elevated preoperative IL-6 (OR $=1.122$; 95\% CI: $0.0619-0.1677, \mathrm{p}=0.029)$ were found to be independent risk factors for NODM. HBV-induced liver cirrhosis, warm ischemia time (WIT), body mass index (BMI), and high preoperative fasting blood glucose (FBG) were also found to be risk factors for NODM. The recipient had a higher risk of NODM if the donor had a high BMI and poor hepatic function. The concentrations of IL-6, procalcitonin (PCT), FBG, and tacrolimus (TAC) in the first month postoperatively were significantly higher in the NODM group than in the NO-NODM group. The survival rate of the patients was not affected by NODM.

Conclusion: HBV-induced liver cirrhosis, WIT, BMI, and high preoperative FBG levels are risk factors for NODM, and age and preoperative IL-6 levels are independent risk factors. To a certain extent, higher BMI and poor hepatic function had reference significance for the incidence of NODM. Patients with a high concentration of FBG, IL-6, and TAC in the first month postoperatively had an increased risk of suffering from NODM.

Keywords: liver transplantation, HBV, IL-6, immunosuppressants, NODM

\section{Introduction}

Since the first liver transplant in 1963 by Professor Starzl et al, liver transplantation (LT) has developed as a unique and effective method of treatment for various endstage liver diseases. It has a success rate of over $90 \%$, a postoperative one-year survival rate of $90 \%$, and a five-year survival rate of approximately $70 \%{ }^{1}$ and has benefited from improvements in surgical techniques, perioperative management, and the introduction and application of immunosuppressants.

However, many clinicians are concerned about complications after LT, especially glucose metabolism disorder. Approximately $30-50 \%$ of patients recovered in a short time without treatment with insulin, and the remaining patients suffered from irreversible new-onset diabetes mellitus (NODM), ${ }^{2}$ which increases the risk of cardiovascular disease, infection, and graft dysfunction and results in higher 
mortality among recipients. The pathogenesis of NODM is identical to type 2 diabetes mellitus (T2DM). Midtvedt et al believed that insulin resistance is its primary pathogenesis, especially in the Impaired Glucose Tolerance (IGT) and Impaired Fasting Glucose (IFG) stages, ${ }^{3}$ but Hur et al suggested that impaired insulin secretion may be the primary mechanism of the development of NODM. ${ }^{4}$ The onset of NODM is correlated with various factors, such as Hepatitis $\mathrm{C}$ virus with a family history of diabetes, elevated BMI, and the administration of immune inhibitors, especially tacrolimus and glucocorticosteroids. ${ }^{5}$

Most of the liver transplant recipients in China suffer from chronic hepatitis B, and some of those have undergone diabetes or impaired glucose tolerance before operation. Hepatitis B virus can cause liver damage and induce glucose metabolism disorders. Some studies have shown that IL-6 can promote the release of inflammatory mediators and have an important impact on the pathogenesis of viral hepatitis. ${ }^{6}$ Luo et al indicated that after the body is infected with HBV, the immune system is activated, inducing hepatocytes to synthesize and secrete IL-6 and IL-8. These cytokines mediate the inflammatory reaction in the liver, promote the chronicity and fibrosis, and exacerbate the disease eventually. ${ }^{7}$

It has been found in animal studies that the hyperosmolar hyperglycemic state can aggravate hepatic ischemia reperfusion injury (HIRI), during which Kupffer cell and macrophage activation can boost the release of inflammatory factors, such as interleukin 1 beta (IL-1 $\beta$ ), tumor necrosis factor alpha (TNF- $\alpha$ ), and IL-6, contributing to apoptosis. ${ }^{8}$ The extended time taken for LT, extensive trauma, intraoperative hemodynamic changes, and HIRI can result in a systemic inflammatory response, which contributes to an increase in the release of cytokines. Over the past few years, studies have demonstrated the relationship between inflammatory factors, insulin resistance, and the subsequent development of T2DM. ${ }^{9}$ Kim et al showed that the excessive release of inflammatory factors is closely associated with obesity, insulin resistance, and T2DM. ${ }^{10}$ Studies by Rotter et al proved that the release of insulin is inhibited by IL6 by inhibiting the activity of PIK3. ${ }^{11}$ The tyrosine phosphorylation of the insulin receptor substrate 1 (IRS-1) blocks the transcription of the insulin signal transduction and downregulates the glucose transporter protein type-4, directly contributing to insulin resistance. ${ }^{12}$ In addition, IL- 6 can reduce the adiponectin level and compete with the signaling pathway of the
Leptin system, decreasing the inhibition of Leptin against insulin secretion and resulting in hyperinsulinemia and insulin resistance. ${ }^{13}$ In vitro studies have shown that significantly inhibiting IL-1 $\beta$ and IL-6 increases glycogen storage in the insulin-stimulated primary culture of rat hepatocytes. ${ }^{14}$ The above evidence suggests that IL-6 may play an important role in NODM.

As a result, this study was primarily designed to analyze the risk factors for NODM, especially the relationship between IL-6 and NODM, to investigate the significance of inflammatory factors in the occurrence of NODM after LT surgery.

\section{Methods and Materials Exclusion Criteria and Data Acquisition}

The present study is a retrospective single-center study. A total of 163 patients who received a liver transplant in our hospital from September 2013 to October 2020 were selected. Exclusion criteria: (1) death within 24 hours after surgery, (2) preoperative diabetes, (3) transplant performed at another center and followed up in our department, (4) less than 18 years old, (5) autologous LT, (6) multi-organ combined transplantation, and (7) follow-up time less than six months. A total of 64 patients were included in this study. The LT surgeries were all performed by the same surgical team using the piggy-back method, and duct-to-duct anastomosis was used for biliary reconstruction. The data collected included patients' general data and laboratory tests within the first month after surgery. At the follow-up visits after discharge, blood glucose, liver function, kidney function, and plasma concentrations were tested. The last follow-up visit was in December 2020.

All patient data relating to the liver transplants were provided by the Liver Transplantation Center of the First Affiliated Hospital of the Xinjiang Medical University and collected by special personnel. All organs were donated voluntarily with written informed consent, and that this was conducted in accordance with the Declaration of Istanbul. The study was subject to the Declaration of Helsinki: Ethical Principles for Medical Research Involving Human Subjects. All procedures performed in studies involving human participants follow the ethical standards developed by the Ethics Committee of The First Affiliated Hospital of Xinjiang Medical University (K202106-01). Written informed consent was obtained from all participants. 


\section{Diagnostic Criteria for NODM}

The diagnostic criteria used in clinical practice are based on the American Diabetes Association's 2003 and the World Health Organization's criteria, ie, the patient should be experiencing symptoms of diabetes mellitus (DM), with a random plasma glucose $\geq 11.1 \mathrm{mmol} / \mathrm{L}$, a fasting plasma glucose $\geq 7.0 \mathrm{mmol} / \mathrm{L}$, or an oral glucose tolerance test two-hour plasma glucose $\geq 11.1 \mathrm{mmol} / \mathrm{L}$. These results were verified by retesting. Once these diagnostic criteria are met, a diagnosis of NODM is established. NODM was defined as patients still requiring insulin for glycemic control after the six-month follow-up visit, under the premise of taking the maintenance dose of immunosuppressants, having a stable graft function, and being free of various acute/chronic complications.

\section{Regimen of Immunosuppressive Drugs}

All patients were treated with a calcineurin inhibitor (TAC or CsA), CellCept (mycophenolate mofetil [MMF]), and hormones as routine immunosuppressive therapy after LT, starting with the administration of TAC at $0.15 \mathrm{mg} / \mathrm{kg} / \mathrm{d}$ or CsA at $5 \mathrm{mg} / \mathrm{kg} / \mathrm{d}$ and MMF at $1500 \mathrm{mg} / \mathrm{d}$ BID within 48 hours after the operation and the intravenous injection of $500 \mathrm{mg}$ methylprednisolone intraoperatively into the portal vein, with subsequent intravenous injection of 240, 200, $160,120,80$ and $40 \mathrm{mg}$ from day one to day seven, respectively. This was then changed to oral administration of prednisone, with an initial dose of $20 \mathrm{mg} / \mathrm{d}$, which was progressively decreased by $5 \mathrm{mg}$ every 10 days and discontinued once the dose reached zero. Cephalosporins were used for early postoperative anti-infection. Within 3-5 days after the operation, antifungal and antiviral drugs were routinely used against infection. Lamivudine or entecavir was orally administered against the recurrence of hepatitis B.

\section{Detection Methods}

\section{Biochemical Index}

Serum urea nitrogen, creatinine, AST, alt, total bilirubin, direct bilirubin and indirect bilirubin were measured by automatic biochemical analyzer (Hitachi 7600, Japan). The kit was purchased from Shanghai Changzheng Co., Ltd. (Shanghai, China).

\section{Blood Glucose}

Blood glucose was measured by glucose oxidase method. The kit was purchased from Shanghai Changzheng Co., Ltd. (Shanghai, China).

\section{Inflammatory Factors}

Serum IL-6 was detected by Luminex 1x-200 analyzer (BD, America). CRP was detected by Purman analyzer. PCT was detected by Roche Cobas E411 electrochemiluminescence analyzer.

\section{Endpoints}

The primary endpoint was the occurrence of postoperative NODM, with patients' survival rate as the secondary endpoint. The survival rate was defined as the time from the first day following the transplant to the date of death from various causes. The last follow-up visit record was used as the endpoint index for patients who were lost to follow-up (LTFU), with LTFU case records and cases without outcome events until the end of the final follow-up visit being defined as censored data. Primary and secondary outcomes after the operation were compared.

\section{Data Analysis Method}

All data were collected using $\mathrm{R}$ language software. The measurement data were expressed as mean \pm standard deviation, and the enumerated data were expressed as numerical values. The normality and homogeneity of variance of the measurement data were tested and further confirmed by the Shapiro-Wilk test. Single-factor analysis measurement data were tested using the $t$-test, and enumerated data using the chi-square test. Variables with $\mathrm{p}<$ 0.05 in the univariate analysis were included in the multivariate logistic regression analysis, and the $95 \%$ CI was recorded. Postoperative survival was analyzed using GraphPad Prism 8 (San Diego, CA), a Kaplan-Meier curve was constructed, and a Log rank test was conducted, and $p<0.05$ was considered to be statistically significant.

\section{Results}

\section{Baseline Data Analysis}

The baseline characteristics of the subjects in the two groups are described in Table 1. Of the 64 patients, 20 (31.25\%) were diagnosed with NODM and 35 (54.67\%) received donation after circulatory death (DCD) transplantation. The univariate analysis revealed that six variables were significantly associated with NODM. The mean age was $52.15 \pm 8.47$ years in the NODM group and $39.27 \pm 11.49$ years in the NO-NODM group ( $p<$ 0.001). Recipients with HBV-induced liver cirrhosis had a higher risk of NODM $(\mathrm{p}<0.001)$. The BMI of patients was $25.8 \pm 4.99$ in the NODM group and $20.4 \pm 3.40$ in 
Table I Clinical Parameters of Liver Transplant Recipients with Diagnosis of NODM and without NODM

\begin{tabular}{|c|c|c|c|}
\hline & NO-NODM & NODM & $\mathbf{P}$ \\
\hline Age(years) & $39.27 \pm 11.49$ & $52.15 \pm 8.47$ & $<0.001$ \\
\hline Gender & & & 0.221 \\
\hline Male & 22 & 14 & \\
\hline Female & 22 & 6 & \\
\hline Donor type & & & 0.053 \\
\hline $\mathrm{DCD}$ & 24 & 5 & \\
\hline LDLT & 20 & 15 & \\
\hline \multicolumn{4}{|l|}{ Etiology } \\
\hline HBV cirrhosis & 21 & 14 & $<0.001$ \\
\hline HCV cirrhosis & 3 & 3 & \\
\hline Cancer & 14 & 2 & \\
\hline Other & 6 & 1 & \\
\hline MELD score & $19.3 \pm 7.68$ & $16.9 \pm 8.45$ & 0.287 \\
\hline Child-Pugh grade & & & 0.139 \\
\hline A & 5 & 5 & \\
\hline B & 19 & 4 & \\
\hline C & 20 & II & \\
\hline Hypertension & 4 & 5 & 0.191 \\
\hline Alcohol consumption & 12 & 4 & 0.756 \\
\hline Smoking history & 13 & 6 & 1 \\
\hline Previous surgery & 12 & 8 & 0.467 \\
\hline $\mathrm{BMI}\left(\mathrm{kg} / \mathrm{m}^{2}\right)$ & $20.4 \pm 3.40$ & $25.8 \pm 4.99$ & $<0.001$ \\
\hline Immunosuppression & & & 0.268 \\
\hline TAC & 30 & 17 & \\
\hline CsA & 14 & 3 & \\
\hline Hospitalization,(days) & $32.9 \pm 10.8$ & $34.4 \pm 16.8$ & 0.706 \\
\hline Follow-up time,(days) & $|296 \pm 76|$ & $1034 \pm 566$ & 0.573 \\
\hline $\mathrm{TC}(\mathrm{mmol} / \mathrm{L})$ & $2.20 \pm 1.34$ & $2.26 \pm 0.624$ & 0.806 \\
\hline Triglycerides $(\mathrm{mmol} / \mathrm{L})$ & I. $12 \pm 0.68$ & $0.874 \pm 0.493$ & 0.115 \\
\hline $\mathrm{HDL}-\mathrm{C}(\mathrm{mmol} / \mathrm{L})$ & $0.684 \pm 0.522$ & $1.12 \pm 2.03$ & 0.359 \\
\hline LDL-C(mmol/L) & $1.42 \pm 0.84$ & $1.24 \pm 0.53$ & 0.308 \\
\hline AST(U/L) & $89.5 \pm 110$ & $172 \pm 334$ & 0.145 \\
\hline $\operatorname{ALT}(\mathrm{U} / \mathrm{L})$ & $85.8 \pm|8|$ & $175 \pm 479$ & 0.283 \\
\hline $\mathrm{DB}$ (umol/L) & $75.9 \pm 97.3$ & $88.7 \pm 91.9$ & 0.622 \\
\hline IB(umol/L) & $69.4 \pm 84.4$ & $82.0 \pm 75.0$ & 0.571 \\
\hline TBIL(umol/L) & $145 \pm 166$ & $169 \pm 158$ & 0.593 \\
\hline $\operatorname{ALB}(g / L)$ & $30.6 \pm 8.80$ & $29.1 \pm 6.52$ & 0.513 \\
\hline
\end{tabular}

(Continued)
Table I (Continued)

\begin{tabular}{|l|c|c|c|}
\hline & NO-NODM & NODM & P \\
\hline FBG(mmol/L) & $5.86 \pm 2.32$ & $8.01 \pm 4.32$ & 0.047 \\
\hline PCT(ng/L) & $1.22 \pm 1.94$ & $8.10 \pm 21.9$ & 0.177 \\
\hline IL-6(pg/mL) & $12.8 \pm 9.67$ & $43.4 \pm 57.9$ & 0.029 \\
\hline CRP(mg/L) & $4.99 \pm 8.19$ & $7.17 \pm 7.50$ & 0.301 \\
\hline Urea(mmol/L) & $5.69 \pm 4.47$ & $4.63 \pm 2.02$ & 0.194 \\
\hline CRE(umol/L) & $65.0 \pm 31.3$ & $60.8 \pm 20.2$ & 0.531 \\
\hline CIT(h) & $2.49 \pm 1.42$ & $3.00 \pm 1.60$ & 0.227 \\
\hline WIT(min) & $4.5 \pm 3.05$ & $7.5 \pm 4.30$ & 0.009 \\
\hline Surgery time(h) & $10.7 \pm 3.17$ & $10.6 \pm 2.05$ & 0.876 \\
\hline Anhepatic stage time(h) & $1.61 \pm 0.76$ & $1.45 \pm 0.57$ & 0.348 \\
\hline Blood loss(mL) & $2655 \pm 1741$ & $2325 \pm 1105$ & 0.365 \\
\hline
\end{tabular}

Abbreviations: LDLT, living donor liver transplantation; DCD, donation after circulatory death; $\mathrm{HBV}$, hepatitis $B$ virus; $\mathrm{HCV}$, hepatitis $\mathrm{C}$ virus; $\mathrm{BMl}$, body mass index; TAC, tacrolimus; TC, total cholesterol; AST, aspartate aminotransferase; ALT, alanine aminotransferase; DB, direct bilirubin; IB, indirect bilirubin; TBIL, total bilirubin; ALB, albumin; FBG, fast blood glucose; PCT, Procalcitonin; IL-6, interleukin-6; CRP, C-reactive protein; CRE, creatinine; CIT, cold ischemia time; WIT, warm ischemia time.

the NO-NODM group $(\mathrm{p}<0.001)$. NODM was more likely to occur if warm ischemia time was prolonged (4.5 \pm 3.05 vs $7.5 \pm 4.30, p=0.008)$. The level of IL- 6 before LT was higher in the NODM group $(43.4 \pm 57.9)$ than in the NO-NODM group $(12.8 \pm 9.67, \mathrm{p}<0.05)$. The FBG level before LT was higher in the NODM group (8.01 \pm 4.32) than in the NO-NODM group $(5.86 \pm 2.32, \mathrm{p}<$ 0.05). Donor-related factors are described in Table 2. The results indicate that recipients of living donor liver transplantation (LDLT) who are obese, male and have a fatty liver, cerebral hemorrhage, or poor liver function have a higher risk of NODM after surgery.

\section{NODM-Related Multivariate Logistic Regression Analysis}

Meaningful indicators from the univariate analysis were included in the multivariate logistic regression analysis. The risk of NODM increased 1.089 times for every extra year of age, as described in Table $3(\mathrm{OR}=1.089$, 95\% CI: $0.0211-0.1495 ; \mathrm{p}=0.003)$. The risk of NODM was 1.122 times higher in subjects with elevated preoperative levels of IL-6 than subjects with NO-NODM IL-6 levels $(\mathrm{OR}=1.122$, 95\% CI: 0.0619-0.1677; $\mathrm{p}=0.029)$. 
Table 2 Clinical and Preoperative Laboratory Test Parameters of DCD \& LDLT Donor

\begin{tabular}{|c|c|c|c|}
\hline & NO-NODM & NODM & $\mathbf{P}$ \\
\hline \multicolumn{4}{|l|}{ DCD } \\
\hline Gender & & & 0.018 \\
\hline Male & 10 & 14 & \\
\hline Female & 10 & I & \\
\hline Age(years) & $36.2 \pm 4.87$ & $37.9 \pm 3.61$ & 0.792 \\
\hline $\operatorname{BMI}\left(\mathrm{kg} / \mathrm{m}^{2}\right)$ & $21.8 \pm 1.40$ & $23.4 \pm 1.39$ & 0.003 \\
\hline Hepatic steatosis & 20 & 15 & 0.005 \\
\hline \multicolumn{4}{|l|}{ Cause of death } \\
\hline Cerebral hemorrhage & II & 15 & 0.011 \\
\hline Cranial trauma & 8 & 0 & \\
\hline Other & I & 0 & \\
\hline Use of vasopressor agents & & & 0.089 \\
\hline Norepinephrine & 16 & 7 & \\
\hline Norepinephrine+Dopamine & 4 & 8 & \\
\hline ICU stay(days) & $16.9 \pm 5.95$ & $17.5 \pm 5.38$ & 0.77 \\
\hline Infection & 20 & 15 & 0.669 \\
\hline AST(U/L) & $31.2 \pm 11.1$ & $49.7 \pm 15.4$ & 0.001 \\
\hline $\operatorname{ALT}(\mathrm{U} / \mathrm{L})$ & $26.6 \pm 11.3$ & $43.2 \pm 13.3$ & 0.001 \\
\hline TBIL (umol/L) & $14.5 \pm 2.76$ & $|7.5 \pm 3.9|$ & 0.018 \\
\hline $\mathrm{Na}+(\mathrm{mmol} / \mathrm{L})$ & $144 \pm 6.56$ & $143 \pm 6.94$ & 0.797 \\
\hline IL-6(pg/mL) & $7.55 \pm 3.06$ & $8.28 \pm 2.72$ & 0.471 \\
\hline \multicolumn{4}{|l|}{ LDLT } \\
\hline Gender & & & I \\
\hline Male & 17 & 4 & \\
\hline Female & 7 & I & \\
\hline Age(years) & $38.8 \pm 6.74$ & $38 \pm 5.24$ & 0.792 \\
\hline $\operatorname{BMI}\left(\mathrm{kg} / \mathrm{m}^{2}\right)$ & $23.1 \pm 1.42$ & $24.7 \pm 1.48$ & 0.003 \\
\hline Hepatic steatosis & 24 & 5 & 0.572 \\
\hline IL-6(pg/mL) & $2.02 \pm 1.50$ & $2.07 \pm 1.58$ & 0.942 \\
\hline
\end{tabular}

Abbreviations: BMI, body mass index; AST, aspartate aminotransferase; ALT, alanine aminotransferase; TBIL, total bilirubin; IL-6, interleukin-6.

The logistic regression equation, Logit $(\mathrm{P})=-16.78+0.83$ $\times \mathrm{X} 1+0.87 \times \mathrm{X} 2+0.211 \times \mathrm{X} 3+0.252 \times \mathrm{X} 4+0.054 \times$ X5 (X1: age, X2: HBV cirrhosis, X3: BMI, X4: FBG, and X5: WIT), was established. The ROC curve (AUC = 0.868, 95\% CI: 0.745-0.992; see Figure 1) was also drawn, and the calibration curve was constructed, proving that the model had a good fit and a small error.
Table 3 Risk Factors of NODM in Multivariate Logistic Regression Analysis

\begin{tabular}{|l|c|c|c|}
\hline & OR & 95\% Cl & P \\
\hline Age(years) & 1.089 & $0.0211-0.1495$ & 0.003 \\
BMI $\left(\mathrm{kg} / \mathrm{m}^{2}\right)$ & 1.235 & $0.0284-0.3931$ & 0.248 \\
HBV cirrhosis & 2.376 & $0.1116-1.6197$ & 0.251 \\
FBG $(\mathrm{mmol} / \mathrm{L})$ & 1.287 & $0.0087-0.4953$ & 0.300 \\
IL-6(pg/mL) & 1.122 & $0.0619-0.1677$ & 0.029 \\
WIT(min) & 1.171 & $0.1037-0.21209$ & 0.731 \\
\hline
\end{tabular}

Abbreviations: BMI, body mass index; FBG, fast blood glucose; IL-6, interleukin-6; WIT, warm ischemia time.

\section{Correlation Between Postoperative Inflammatory Factors and NODM}

The IL-6, CRP, and PCT levels were measured on days 1, 4, $7,14,21$, and 28 postoperatively and compared between the groups. On days 1, 7, 14, 21 and 28 postoperatively, the IL-6 levels were significantly higher in the NODM group than in the NO-NODM group ( $<<0.05$; see Figure $2 \mathrm{~A})$. On days 1 , 4,21 , and 28 postoperatively, the PCT levels were significantly higher in the NODM group than in the NO-NODM group ( $<<0.05$; see Figure 2B). There was no significant difference in the CRP levels between the two groups at any of the time points $(p<0.05$; see Figure $2 C$ ).

\section{Correlation of TAC Plasma Concentration and NODM}

TAC is a well-recognized risk factor for NODM. On days 16, 21, and 28 postoperatively, the TAC concentrations were significantly higher in the NODM group than in the NO-NODM group ( $<<0.05$; see Figure 3$)$.

\section{Correlation of Postoperative FBG and NODM}

In the first month postoperatively, the FBG levels were significantly higher in the NODM group than in the NONODM group ( $\mathrm{p}<0.05$; see Figure 4$)$.

\section{Correlation Between NODM and Outcome}

In the NO-NODM group, 11 patients (25\%) died, with the follow-up time being $1296 \pm 761$. In the NODM group, 4 patients (20\%) died, with the follow-up time being $1034 \pm$ 566. The Kaplan-Meier survival curve was constructed, and the Log rank test was performed, and both showed 


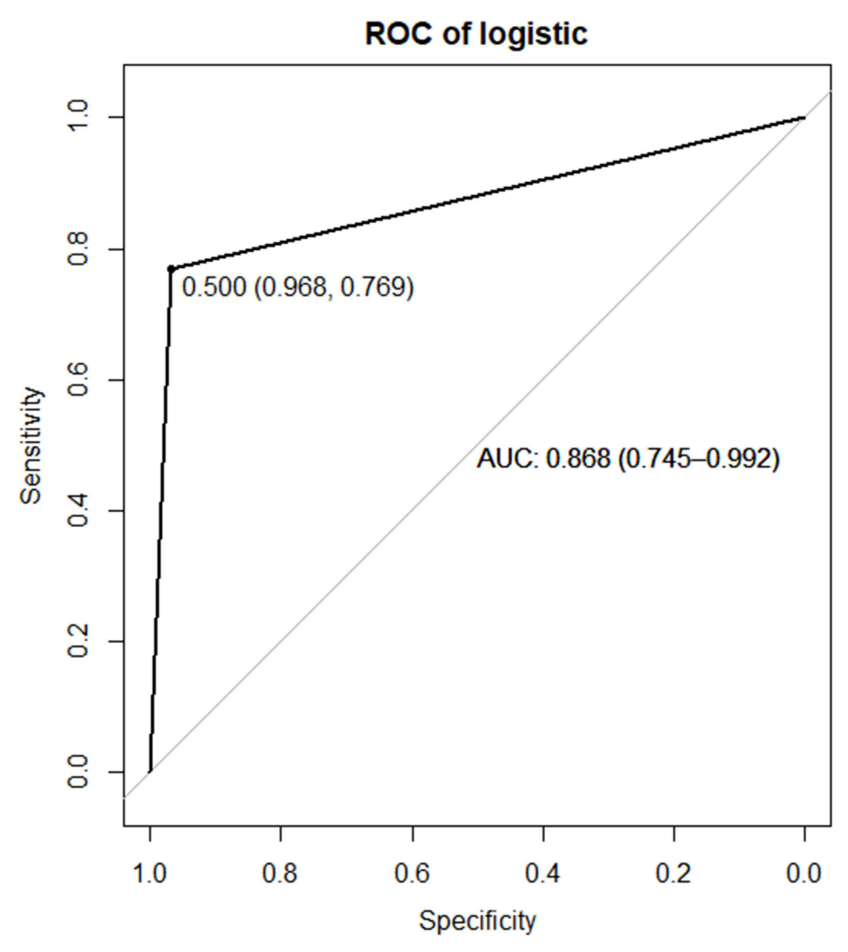

Figure I Constructed Logistic regression model was used to predict the test set and construct the ROC chart. It can be seen from the figure that $A U C=0.868,95 \%$ $\mathrm{Cl}: 0.745,0.992$, indicating that the prediction effect of the model is excellent.

that there was no significant difference in patient survival between the two groups $(\mathrm{p}=0.973$; see Figure 5).

\section{Discussion}

The results of this study confirmed that IL-6 is a risk factor for NODM. Previous studies have shown a strong correlation between IL-6 levels, insulin resistance, and T2DM, ${ }^{15}$ but there have been no reports on the correlation between NODM and IL-6 levels. As a result, we have broken new ground with this study. The main findings of this study were as follows: (1) BMI, warm ischemia time, and elevated preoperative blood glucose levels are risk factors for NODM, while age and high preoperative IL-6 levels are independent risk factors for NODM. (2) High IL-6 and FBG levels and TAC plasma concentration in the first month postoperatively are risk factors for NODM. (3) NODM has no significant effect on the long-term survival rate of patients.

This study revealed that patients with high preoperative IL-6 levels had a higher incidence of NODM $(\mathrm{OR}=$ 1.122, 95\% CI: $0.0619-0.1677 ; \mathrm{p}=0.029)$. It is possible that these patients already had preoperative insulin resistance caused by chronic inflammation. In addition, even in the presence of confounders, such as the use of antibiotics and individual differences in patient characteristics, at days $1,7,14,21$, and 28 postoperatively, the IL-6 concentrations were significantly higher in the NODM group than in the NO-NODM group. IL-6 is normally present in all tissues, but it can lead to inflammation, which further leads to insulin resistance and T2DM when it is produced irregularly and expressed abundantly. $^{9}$ IL-6, a pro-inflammatory cytokine, can induce cytokine signaling 3, resulting in the inhibition of the transduction of insulin signals. IL- 6 receptors are related to the cytokine class I receptor family and can activate the transcription factor JAK/STAT signaling pathway to induce STAT phosphorylation to regulate the transcription of target genes. ${ }^{16}$ Kanemaki et al
A

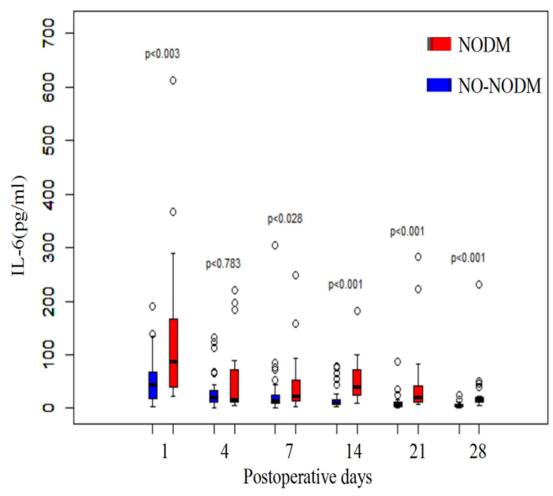

B

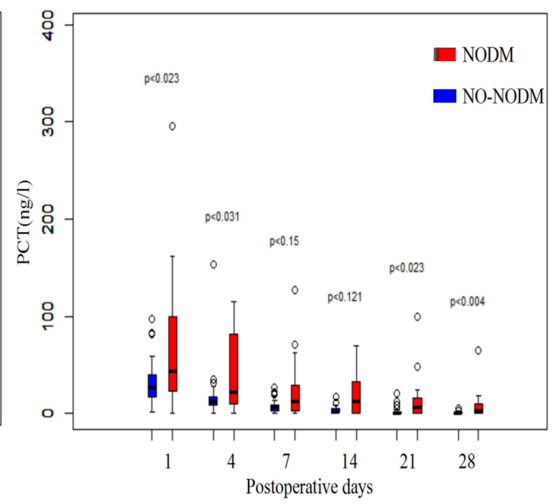

C

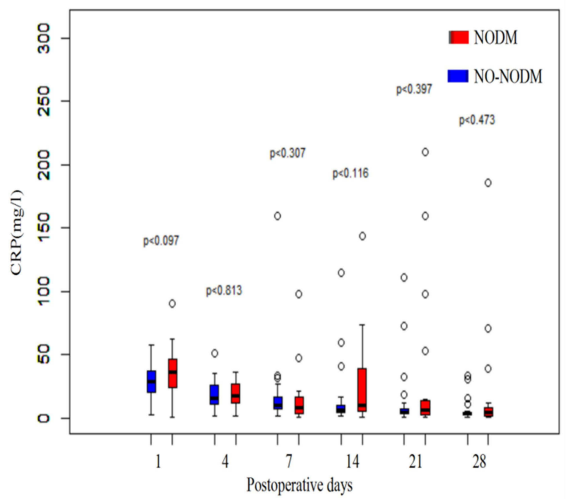

Figure 2 (A) The IL-6 levels of NO-NODM group in NODM combination after liver transplantation, the inner lines represent the median, the bars represent the $25 \%$ and $75 \%$ confidence intervals, and the error bars represent the $5 \%$ and $95 \%$ confidence intervals. (B) The PCT levels of NO-NODM group in NODM combination after liver transplantation, the inner lines represent the median, the bars represent the $25 \%$ and $75 \%$ confidence intervals, and the error bars represent the $5 \%$ and $95 \%$ confidence intervals. (C) The CRP levels of NO-NODM group in NODM combination after liver transplantation, the inner lines represent the median, the bars represent the $25 \%$ and $75 \%$ confidence intervals, and the error bars represent the $5 \%$ and $95 \%$ confidence intervals. 


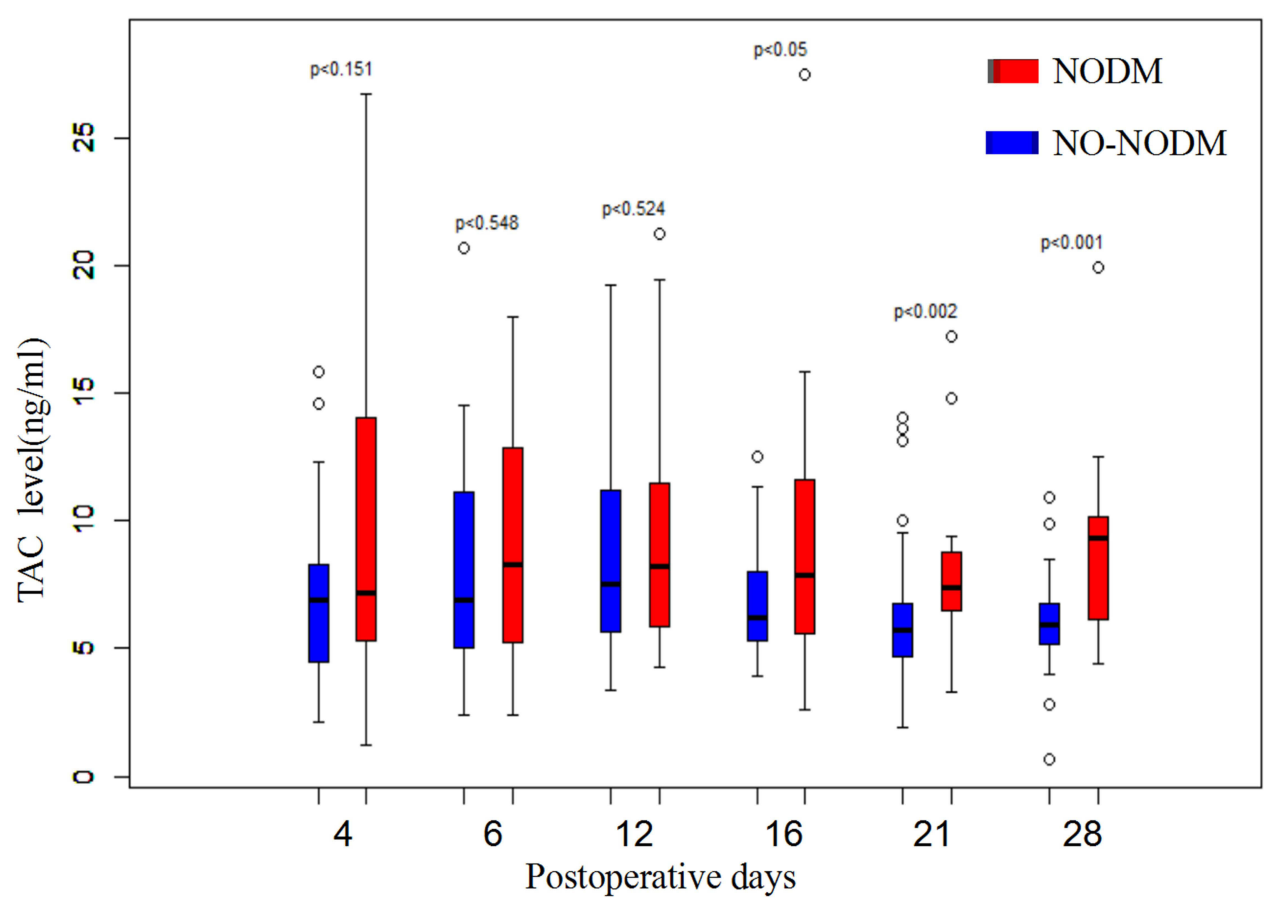

Figure 3 The TAC plasma concentration of NO-NODM group in NODM combination after liver transplantation, the inner lines represent the median, the bars represent the $25 \%$ and $75 \%$ confidence intervals, and the error bars represent the $5 \%$ and $95 \%$ confidence intervals.

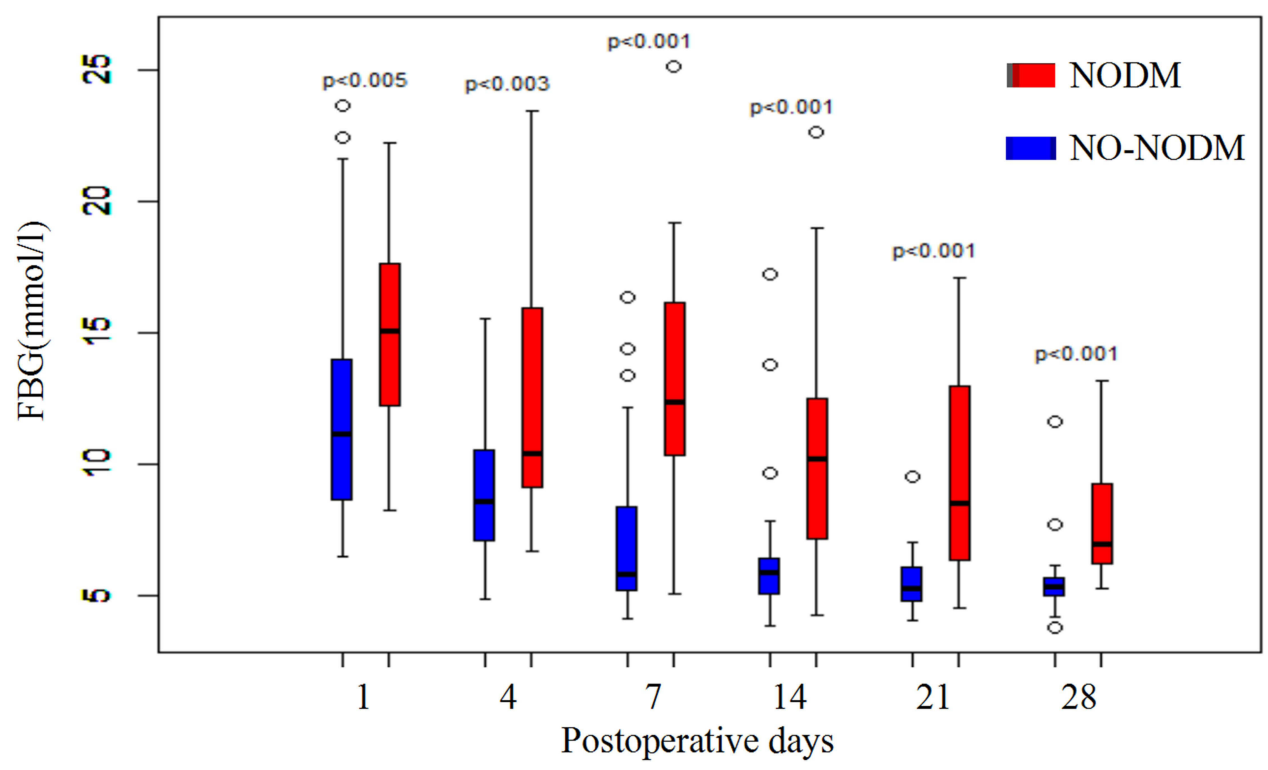

Figure 4 The FBG levels of NO-NODM group in NODM combination after liver transplantation, the inner lines represent the median, the bars represent the $25 \%$ and $75 \%$ confidence intervals, and the error bars represent the $5 \%$ and $95 \%$ confidence intervals.

showed that under the pathological state, the liver glucose metabolism was preferentially controlled by cytokines (IL-1 $\beta$ and IL-6) rather than glucose regulatory hormones, further leading to the occurrence of insulin resistance. $^{14}$ Insulin resistance and inflammatory response interacted with and aggravated each other.
Blocking the related inflammatory response pathways may be an effective strategy for the prevention and treatment of NODM.

Age is a well-recognized risk factor. Kuo et al showed, in a large retrospective study with a sample size of 20,172 , that age $\geq 50$ years is an independent risk factor for 


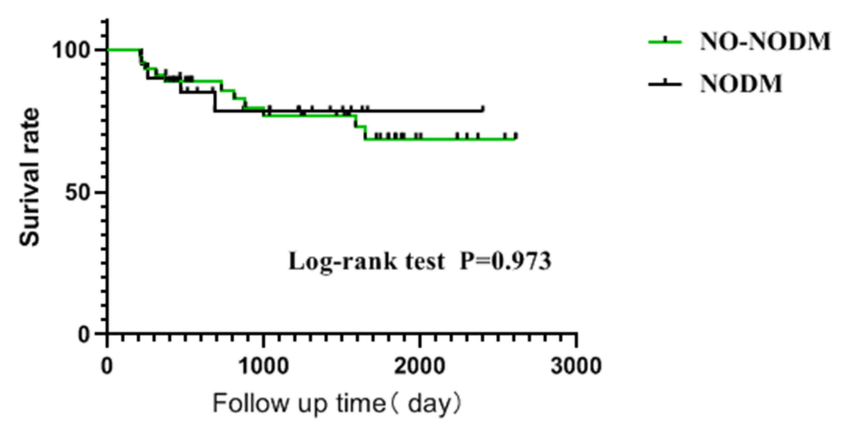

Figure 5 Kaplan-Meier survival curve analysis of 2 group.

NODM. ${ }^{17,18}$ The present study demonstrated that the risk of NODM was increased 1.089 times (OR $=1.089 ; 95 \%$ CI: $0.0211-0.1495, p=0.003$ ) for each one-year increase in age.

In this study, univariate analysis revealed that HBV liver cirrhosis is a risk factor for postoperative blood glucose abnormalities. Some studies have shown that the failed liver has a direct "toxic" effect on $\beta$ cells. ${ }^{19}$ The specific reasons for this effect still need to be investigated, but it is important to improve the treatment of $\beta$-cell functional maintenance in patients with liver cirrhosis. The insulin concentration in the portal vein is $2-3$ times higher than in the peripheral vein, and the portal vein contains approximately $60 \%$ of the insulin secreted by the pancreas. Most of this insulin is removed in the liver, as an initial pathway. ${ }^{20,21}$ In the case of liver failure, the ability of the liver to remove insulin decreases, which results in insulin resistance. ${ }^{9}$ This may be the mechanism of postoperative NODM in patients with HBV liver cirrhosis. Patients with high preoperative blood glucose levels who have not been diagnosed with DM may have hepatogenic hyperglycemia. The research of Li confirmed that the high fasting blood glucose before operation was significantly correlated with the occurrence of NODM after operation. In our study, the incidence of postoperative NODM in these patients was higher than in patients with normal preoperative blood glucose levels $(\mathrm{p}<0.05)$, which was related to both liver dysfunction and $\beta$-cell dysfunction.

Hartog et al confirmed that HIRI affected IRS-1, IRS2, and phosphoinositide 3-kinase to induce insulin resistance and was related to graft dysfunction, which was a risk factor for the occurrence of NODM in the early postoperative period. ${ }^{23}$ This study confirmed that the longer the duration of WIT, the greater the risk of NODM. The ischemia-reperfusion injury was essentially attributed to the liver injury and the release of inflammatory factors. Ilmakunnas et al confirmed that the release of large amounts of high mobility group box 1 protein (HMGB1) from the inferior vena cava after LT in humans is an important marker of liver injury. ${ }^{24}$ As a member of the damage-associated molecular patterns family, HMGB1 is an important pro-inflammatory factor that can induce the release of inflammatory factors, such as IL- $1 \beta$, IL-6, and TNF- $\alpha$, thereby aggravating liver injury. ${ }^{25}$ HMGB1 can be also combined with IL-1 to promote pancreatic islet inflammation and $\beta$-cell death. Multiple studies on T2DM have confirmed that HMGB1 participates in the development of insulin resistance by activating the NF$\kappa \mathrm{B}$ signaling pathway and promoting an increase in the expression of inflammatory mediators. ${ }^{26}$ Insulin resistance after LT may be related to HMGB1, and studies on HMGB1-related pathways may provide new ideas for the prevention and treatment of NODM.

In this study, it was found that higher BMI was an independent risk factor for NODM, and many studies have confirmed that a BMI $>30 \mathrm{~kg} / \mathrm{m} 2$ can result in an increase in the occurrence of cardiovascular diseases. ${ }^{22,27}$ Ling et al conducted a study on the China Liver Transplant Database and found that the incidence rate of New Onset Diabetes after Transplantation (NODAT) was 1.25 times higher when BMI was $\geq 25 \mathrm{~kg} / \mathrm{m} 2$ than when it was $<25 \mathrm{~kg} /$ $\mathrm{m}^{2}{ }^{28}$ However, subjects with end-stage liver disease were complicated by ascites and malnutrition, which may affect the results of the study. For obese patients, it is necessary to reduce weight and abdominal circumference as much as possible before surgery to decrease the incidence of NODM.

In this study, the standard postoperative immunosuppressant regimen for most recipients $(n=47)$ was TAC. The use of TAC had no predictive significance for the incidence of postoperative NODM, but the plasma concentration one month after surgery was statistically significantly higher in the NODM group than the NO-NODM group. It was further verified that TAC, as a calcineurin inhibitor, resulted in insulin resistance and further led to NODM in recipients. Yagi et al confirmed that a TAC plasma concentration $\geq 8 \mathrm{ng} / \mathrm{mL}$ three months after LDLT surgery is an independent risk factor for NODM. ${ }^{29}$ Ana confirmed that the toxicity of TAC dependent on insulin resistance caused by the existing $\beta$-cell dysfunction. ${ }^{30}$ Transcription factors play an important role in the production and proliferation of insulin, and TAC may not have a toxic effect if insulin resistance did not already exist. ${ }^{30}$ 
Rostaing et al confirmed that the effect of tacrolimus sustained-release preparations is equivalent to the regular forms. $^{31}$ Therefore, in the case of the same immunosuppressive effect, the use of the immunosuppressive therapy regimen that has the least impact on human glucose metabolism may reduce the incidence of NODM.

This study showed that the rate of occurrence of NODM was lower in recipients of LDLT (17.24\%) than those of DCD (42.86\%). However, this difference was not statistically significant. The intra-group comparison revealed that the higher the BMI of the donor, the greater the risk of postoperative NODM. In DCD donors, the risk of NODM was higher from donors with cerebral hemorrhage surgery, donors with elderly liver, donors with steatosis and donors with hepatic insufficiency. However, the sample size was small during intra-group comparison, which could lead to bias. Donor type and gene polymorphism were associated with an increased risk of NODM in recipients. ${ }^{32-34}$ In the era of DCD liver donation, regulating the glucose metabolism in the recipient and reducing the incidence of NODM are problems that still need to be solved.

This study had several limitations. Xinjiang is in northwest China, and our hospital is the only one in Xinjiang that is qualified to perform LT. The sample size was small, and the retrospective study suffered from recall and information bias. Therefore, we shared our data in as much detail as possible with the hopes of enriching the information in the LT database and providing new ideas for future studies on the prevention of NODM.

\section{Conclusion}

NODM is a common complication after LT, and its occurrence is related to many factors. According to this study, patients with advanced age, hepatitis B cirrhosis, high preoperative levels of IL-6, high preoperative FBG levels, high BMI, and long warm ischemic times had a higher risk of developing NODM. Patients with high levels of IL-6, FBG, and TAC in the first month postoperatively were at an increased risk of developing NODM later. Recipients who received livers from patients with a high BMI and liver dysfunction had a higher risk of NODM after surgery, and strict follow-up was required. It is suggested that a prospective, multi-center collaborative cohort study should be carried out to further investigate the nature of and risk factors for NODM. It is significant for clinical practice to conduct a comprehensive assessment of the high-risk factors for NODM before surgery by understanding its pathogenesis to enable early intervention and the formulation of individualized treatment measures to reduce the incidence of NODM and improve the longterm survival rate and quality of life of patients.

\section{Ethics Approval and Consent to Participate}

This study was conducted with approval from the Ethics Committee of The First Affiliated Hospital of Xinjiang Medical University (K202106-01). This study was conducted in accordance with the declaration of Helsinki. Written informed consent was obtained from all participants.

\section{Acknowledgments}

We would like to acknowledge the hard and dedicated work of all the staff that implemented the intervention and evaluation components of the study.

\section{Funding}

This study was sponsored by the Natural Science Foundation of Xinjiang (No.2021D01C353).

\section{Disclosure}

The authors declare that they have no competing interests.

\section{References}

1. Samuel D, Coilly A. Management of patients with liver diseases on the waiting list for transplantation: a major impact to the success of liver transplantation. BMC Med. 2018;16(1):113. doi:10.1186/s12916-0181110-y

2. Ahn HY, Cho YM, Yi NJ. Predictive factors associated with the reversibility of post-transplantation diabetes mellitus following liver transplantation. J Korean Med Sci. 2009;24(4):567-570. doi:10.3346/ jkms.2009.24.4.567

3. Midtvedt K, Hjelmesaeth J, Hartmann A, et al. Insulin resistance after renal transplantation: the effect of steroid dose reduction and withdrawal. Am Soc Nephrol. 2003;15:3233-3239. doi:10.1097/01. ASN.0000145435.80005.1E

4. Hur KY, Kim MS, Kim YS, et al. Risk factor associated with the onset and progression of post transplantation diabetes in renal allograft recipients. Diabetes Care. 2007;19:136-143.

5. Jenssen T, Hartmann A. Post-transplant diabetes mellitus in patients with solid organ transplants. Nat Rev Endocrinol. 2019;15(3):172-188. doi:10.1038/s41574-018-0137-7

6. Chen Z, Li YX, Fu HJ, et al. Hepatitis B virus core antigen stimulates IL-6 expression via p38, ERK and NF- $\mathrm{KB}$ pathways in hepatocytes. Cell Physiol Biochem. 2017;41(1):91-100. doi:10.1159/000455954

7. Luo HB, Xia W, Cao HT, et al. Expression and clinical significance of T cell subsets, IL-6 and IL-8 levels in peripheral blood of patients with hepatitis B. Prog Mod Biomed. 2018;18(7):2517-2521.

8. Chen CF, Leu FJ, Chen HI, et al. Lack of a protective effect of insulin on three reperfusion-liver injury models in rats and mice. Transplant Proc. 2006;38(7):2221-2225. doi:10.1016/j.transproceed.2006.07.016

9. Rehman K, Akash MSH, Liaqat A, Kamal S, Qadir MI, Rasul A. Role of interleukin-6 in development of insulin resistance and type 2 diabetes mellitus. Crit Rev Eukaryot Gene Expr. 2017;27(3):229-236. doi:10.1615/CritRevEukaryotGeneExpr.2017019712 
10. Kim KE, Heo JS, Han S, et al. Blood concentrations of lipopolysaccharide-binding protein, high-sensitivity C-reactive protein, tumor necrosis factor- $\alpha$, and interleukin- 6 in relation to insulin resistance in young adolescents. Clin Chim Acta. 2018;486:115-121. doi:10.1016/j.cca.2018.07.042

11. Rotter V, Nagaev I, Smith U. Interleukin.6(IL.6) induces insulin resistance in 333-L1 adipocytes and is, like IL-8 and tumor necrosis factor-alpha, over expressed in human fat cells from insulin-resistant subjects. $J$ Biol Chem. 2003;278(46):45777-45784. doi:10.1074/jbc.M301977200

12. Fève B, Bastard JP. The role of interleukins in insulin resistance and type 2 diabetes mellitus. Nat Rev Endocrinol. 2009;5(6):305-311. doi:10.1038/nrendo.2009.62

13. Fasshauer M, Kralisch S, Klier M, et al. Adiponectin gene expression and secretion is inhibited by interleukin-6 in 3T3-L1 adipocytes. Biochem Biophys Res Commun. 2003;301(4):1045-1050. doi:10. 1016/S0006-291X(03)00090-1

14. Kanemaki $\mathrm{T}$, Kitade $\mathrm{H}$, Kaibori $\mathrm{M}$, et al. Interleukin $1 \beta$ and interleukin 6 , but not tumor necrosis factor $\alpha$, inhibit insulin-stimulated glycogen synthesis in rat hepatocytes. Hepatology. 1998;11 (27):1296-1303. doi:10.1002/hep.510270515

15. Pradhan AD, Manson JE, Rifai N, Buring JE, Ridker PM. C-reactive protein, interleukin 6 , and risk of developing type 2 diabetes mellitus. JAMA. 2001;2863(3):327-334. doi:10.1001/jama.286.3.327

16. Bastard J-P, Maachi M, Lagathu C, et al. Recent advances in the relationship between obesity, inflammation, and insulin resistance. Eur Cytokine Netw. 2006;17(1):4-12.

17. Kuo H-T, Sampaio MS, Ye X, et al. Risk factors for new-onset diabetes mellitus in adult liver transplant recipients, an analysis of the organ procurement and transplant network/united network for organ sharing database. Transplantation. 2010;89(9):1134. doi:10. 1097/TP.0b013e3181d2fec1

18. Zheng J, Wang WL. Risk factors of metabolic syndrome after liver transplantation. Hepatobiliary Pancreat Dis Int. 2015;14(6):582-587. doi:10.1016/S1499-3872(15)60037-6

19. Orsi E, Grancini V, Menini S, Aghemo A, Pugliese G. Hepatogenous diabetes: is it time to separate it from type 2 diabetes? Liver Int. 2017;37(7):950-962. doi:10.1111/liv.13337

20. Hui JM, Sud A, Farrell GC, et al. Insulin resistance is associated with chronic hepatitis $\mathrm{C}$ virus infection and fibrosis progression. Gastroenterology. 2003;125:1695-1704. doi:10.1053/j.gastro.2003.08. 032

21. Grancini V, Trombetta M, Lunati ME, et al. Central role of the beta-cell in driving regression of diabetes after liver transplantation in cirrhotic patients. J Hepatol. 2019;5(70):954-962. doi:10.1016/j. jhep.2019.01.015

22. Li DW, Lu TF, Hua XW, et al. Risk factors for new onset diabetes mellitus after liver transplantation: a meta-analysis. World J Gastroenterol. 2015;21(20):6329-6340. doi:10.3748/wjg.v21.i20.6329
23. Hartog H, May CJ, Corbett C, et al. Early occurrence of new-onset diabetes after transplantation is related to type of liver graft and warm ischaemic injury. Liver Int. 2015;35(6):1739-1747. doi:10.1111/ liv. 12706

24. Ilmakunnas M, Tukiainen EM, Rouhiainen A, et al. High mobility group box 1 protein as a marker of hepatocellular injury in human liver transplantation. Liver Transpl. 2008;14(10):1517-1525. doi:10.1002/lt.21573

25. Huebener P, Pradere JP, Hernandez C, et al. The HMGB1/RAGE axis triggers neutrophil-mediated injury amplification following necrosis. J Clin Invest. 2015;125(2):539-550. doi:10.1172/JCI76887

26. Pahwa R, Jialal I. The role of the high-mobility group box 1 protein-toll like receptor pathway in diabetic vascular disease. $J$ Diabetes Complications. 2016;30(6):1-6. doi:10.1016/j. jdiacomp.2016.03.005

27. Hryniewiecka E, Zegarska J, Paczek L. Arterial hypertension in liver transplant recipients. Transplant Proc. 2011;43(8):3029-3034. doi:10.1016/j.transproceed.2011.07.011

28. Ling Q, Xu X, Xie H, et al. New-onset diabetes after liver transplantation: a national report from China liver transplant registry. Liver Int. 2016;36(5):705-712. doi:10.1111/liv.13042

29. Yagi S, Kaido T, Iida T, et al. New-onset diabetes mellitus after living-donor liver transplantation: association with graft synthetic function. Surg Today. 2017;47:733-742. doi:10.1007/s00595-0161444-z

30. Rodriguez-Rodriguez AE, Donate-Correa J, Rovira J, et al. Inhibition of the mTOR pathway: a new mechanism of $\beta$ cell toxicity induced by tacrolimus. Am J Transplant. 2019;4(19):3240-3249. doi:10.1111/ ajt. 15483

31. Rostaing L, Bunnapradist S, Grinyó JM, et al. Novel once-daily extended-release tacrolimus versus twice-daily tacrolimus in de novo kidney transplant recipients: two-year results of phase 3, double-blind, randomized trial. Am J Kidney Dis. 2016;67 (4):648-659. doi:10.1053/j.ajkd.2015.10.024

32. Grancini V, Resi V, Palmieri E, Pugliese G, Orsi E. Management of diabetes mellitus in patients undergoing liver transplantation. Pharmacol Res. 2019;1(141):556-573. doi:10.1016/j.phrs.2019.01. 042

33. Yadav AD, Chang YH, Aqel BA, et al. New onset diabetes mellitus in living donor versus deceased donor liver transplant recipients: analysis of the UNOS/OPTN database. J Transplant. 2013;20 13:269096. doi: $10.1155 / 2013 / 269096$

34. Ling Q, Xie H, Lu D, et al. Association between donor and recipient TCF7L2 gene polymorphisms and the risk of new-onset diabetes mellitus after liver transplantation in a Han Chinese population. J Hepatol. 2013;58(2):271-277. doi:10.1016/j.jhep.20 12.09.025
International Journal of General Medicine

\section{Publish your work in this journal}

The International Journal of General Medicine is an international, peer-reviewed open-access journal that focuses on general and internal medicine, pathogenesis, epidemiology, diagnosis, monitoring and treatment protocols. The journal is characterized by the rapid reporting of reviews, original research and clinical studies across all disease areas. The manuscript management system is completely online and includes a very quick and fair peer-review system, which is all easy to use. Visit http://www.dovepress.com/ testimonials.php to read real quotes from published authors. 